\title{
Multiple Sclerosis and Clinical Gait Analysis before and after Fampridine: A Systematic Review
}

\author{
Magaly Lecat ${ }^{a, b}$ Pierre Decavel ${ }^{a, c}$ Eloi Magnin ${ }^{c, d}$ Brigitte Lucas ${ }^{b}$ \\ Vincent Gremeaux ${ }^{e}$ Yoshimasa Sagawa ${ }^{a, c}$ \\ aLaboratory of Clinical Functional Exploration of Movement, University Hospital of Besançon, Besançon, France; \\ ${ }^{b}$ Functional Rehabilitation Center Divio, Dijon, France; ${ }^{C}$ Integrative and Clinical Neurosciences EA481, Bourgogne \\ Franche-Comte University, Besançon, France; 'Department of Neurology, University Hospital of Besançon, \\ Besançon, France; ${ }^{e}$ Department of Rehabilitation, University Hospital of Dijon, Dijon, France
}

\section{Keywords}

Multiple sclerosis · Gait · Fampridine · Long term

\begin{abstract}
Background: Gait impairment is one of the most disabling symptoms in people with multiple sclerosis (PwMS). Fampridine, has demonstrated a positive effect on gait speed in PwMS after 14 days of treatment but the long-term effects have not yet been demonstrated. This study reviews the long-term effects of fampridine on gait in PwMS. Summary: This systematic review was conducted according to the PRISMA statement. Studies were considered long term if treatment exceeded 28 days. From the 498 studies identified, 18 $(2,200$ patients) fulfilled all eligibility criteria. Only 3 studies followed-up patients for $>1$ year and one of these showed a non-significant improvement in the gait speed. Key Messages: Fampridine seems to be beneficial at improving gait speed in PwMS in the long term. Further long-term studies are needed on related gait and functional parameters.
\end{abstract}

(c) 2017 S. Karger AG, Basel
๑ 2017 S. Karger AG, Basel

E-Mail karger@karger.com

www.karger.com/ene

\section{Introduction}

Multiple sclerosis (MS) is a chronic progressive inflammatory disease of the central nervous system. Early in the course of MS, patients are frequently affected by gait disorders resulting from numerous symptoms, especially muscle weakness, balance disorders, coordination disorders, spasticity, or impaired epicritic sensitivity. Studies on patients with MS show that almost half report impaired gait: notably, a decrease in walking speed and distance $[1,2]$, and this was reported as the most disabling symptom of MS in $70 \%$ of cases $[1,3]$. Walking is an essential aspect of many everyday activities; its impairment can diminish quality of life [4] and lead to economic and social difficulties [5]. All of these considerations highlight the interest in studying gait disorders and managing them as effectively as possible.

Until recently, there was no medical treatment that could specifically improve walking abilities in patients with MS, whatever the cause of the impairment. Fampridine (4-aminopyridine) is the first symptomatic treat- 
ment specifically indicated for patients with MS who present with gait disorders [6]. Fampridine is a potassium-channel inhibitor; it reduces ionic currents through channels, thus prolonging repolarization and improving the formation of action potentials in demyelinated axons [7]. By improving the formation of action potentials, fampridine can improve conduction within the central nervous system, notably in the motor system [7]. This drug has been marketed in the Unites States since 2010 and obtained marketing approval in France in 2011.

Phase I and II therapeutic trials have shown a therapeutic effect of fampridine on gait, by increasing walking speed, measured over a distance of 25 feet $(7.62 \mathrm{~m})$ by $\sim 25 \%[8,9]$. Patients who presented with a clinically objective improvement were considered to be responders and those with no improvement were considered as nonresponders.

The short-term effects of fampridine are well-documented whereas little is known about its long-term effects. It is therefore important to determine whether or not treatment is efficacious in the longer term and to assess disease progression as well as tolerance to treatment.

The aim of this systematic review was to evaluate published studies on the effects of short- and long-term treatment with fampridine on the gait of persons with MS, as well any adverse effects from fampridine.

\section{Methods}

\section{Assessment for Inclusion into the Study}

We conducted a systematic review of the literature using PRISMA methodology $[10,11]$. All articles were read by 2 readers, and a third reader was called upon when necessary. The review grid (3.1.4) was followed and validated by consensus from the 3 readers.

Step 1: Identification of Pertinent Studies

To identify the relevant articles, the following keywords and operators were typed - "multiple sclerosis" AND "fampridine" OR "4 aminopyridine" OR "Fampyra" - into the 3 main medical databases: PubMed, Embase, and Cochrane. Given the relatively recent availability of this drug, the search was conducted with no time limits.

Step 2: Selection of Articles

Duplicate articles were excluded according to their titles. The titles were then read and any articles with titles containing the terms "systematic review" and/or "pharmacology" and/or "animals" were excluded, as were articles in languages other than English.

Step 3: Eligibility for Inclusion

The following inclusion criterion was added: study of gait in patients with MS treated with fampridine. If the abstracts did not refer at least partially to this criterion, the articles were excluded.

MS and Clinical Gait Analysis before and after Fampridine
As for the type of article, reviews of articles that did not correspond to a clinical trial were excluded, as were articles not available in their entirety.

Step 4: Inclusion of Articles

During this step, articles were read in their entirety and studies that did not include a quantitative analysis of gait were excluded as were preliminary studies. Articles mentioned in the reference sections of included articles were also analyzed using the inclusion steps mentioned above. Articles that met all of the selection criteria were separated into 2 arbitrarily determined categories according to study duration: that is, short-term ( $\leq 28$ days) or long-term ( $>28$ days).

Each article was classified according to its level of scientific evidence (LE). LE ranged from 1 to 4: 1 corresponded to high-power randomized controlled trials; 2 corresponded to low-power randomized controlled trials, to non-randomized controlled studies, or to cohort studies; 3 corresponded to case-control studies; and 4 corresponded to controlled studies with a major bias (selection bias, absence of randomization,...), retrospective studies, and case series.

\section{Results}

\section{Selection of Articles}

Figure 1 shows the different steps followed in this review, from identifying to the inclusion of selected studies, as well as the various reasons for exclusion of any articles in these steps.

In the identification step, 498 articles were found. In the selection step, after reading the title, 115 duplicates were excluded, leaving 383 articles (online suppl. material, see www.karger.com/10.1159/000480729). Among these studies, 111 dealt with the pharmacology of fampridine, 84 dealt exclusively with MS, 81 were outside the topic, and 14 were animal trials. Altogether, 310 articles were excluded and 73 articles were eligible.

In the eligibility step and after reading the Abstracts, 21 articles did not include a study of walking and 11 were reviews of the literature. Altogether, 51 articles were excluded and 22 articles met the inclusion criteria.

For the inclusion step, 22 articles were read in their entirety. A total of 17 articles conducted a quantitative analysis of walking. After reading the reference section of these articles, 1 additional article was included. Altogether, 18 articles were included. According to the predetermined cutoff period (28 days), 5 articles carried out short-term analyses and 13 articles conducted long-term analyses. All articles that carried out long-term analyses also carried out shortterm analyses, which were taken into account in the systematic review (i.e., 18 articles with short-term analyses).

Using the review grill proposed by HAS, 7 studies were considered LE 1 and 8 as LE 2. These were multicenter, 


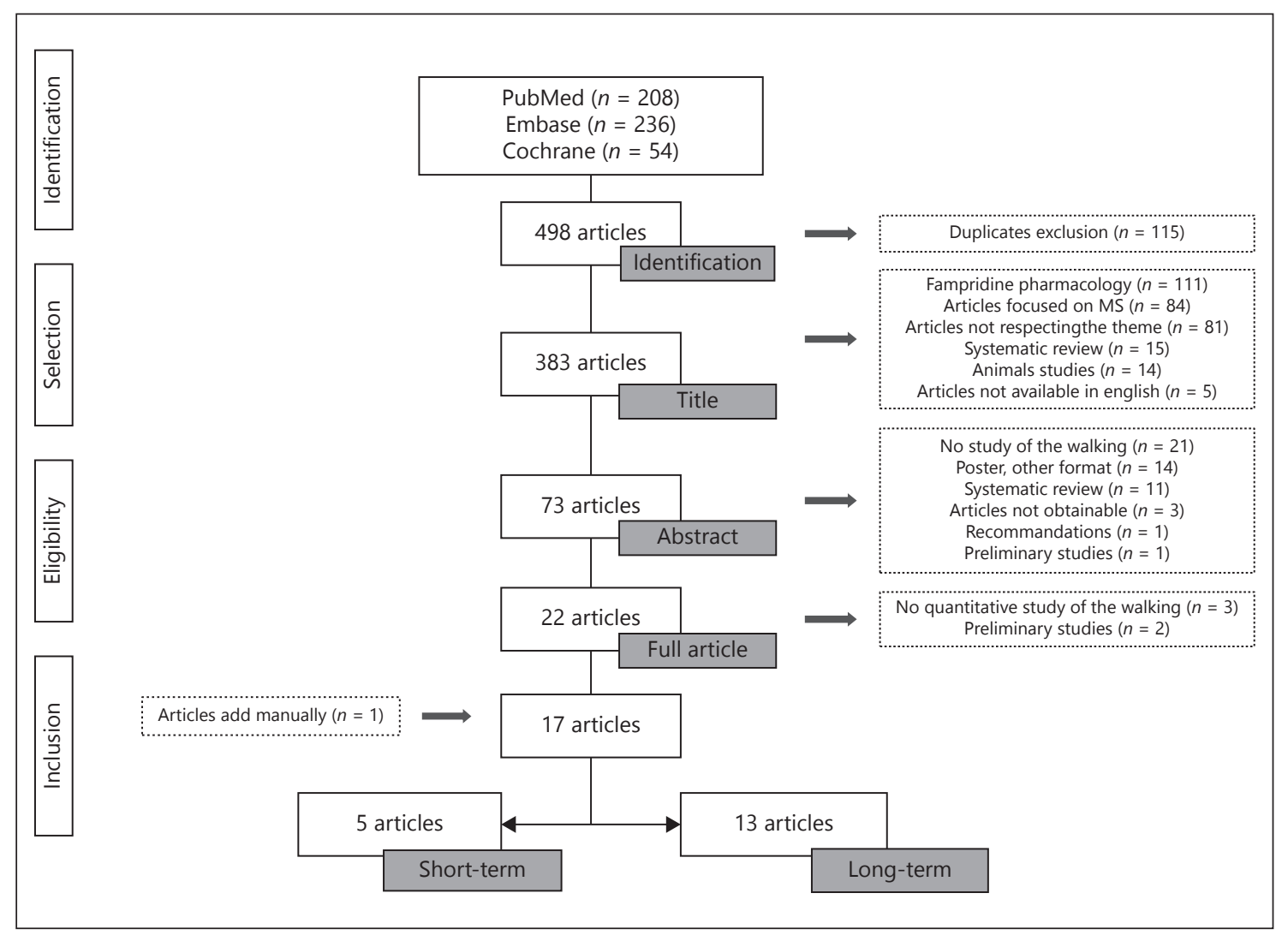

Fig. 1. Study selection flow chart.

randomized, double-blind, placebo-controlled studies. Given the small number of articles, they were analyzed independently of their LE; the results must therefore be interpreted with caution.

The references for all of the studies excluded from this systematic review are listed in the Annex.

\section{Choice of Participants}

As well as the diagnosis of MS, certain inclusion criteria were common to all of the studies. All forms of MS were included (relapsing remitting, primary progressive, secondary progressive) [12]. There were wide variations in terms of age, ranging from 18 to 70 years $[8,13-16]$ or from 18 to 60 years $[17,18]$.

The Expanded Disability Status Scale (EDSS) was not always referred to in the inclusion criteria. When it was taken into account, the score had to be between 4 and 7 inclusive [17-22] or between 3.5 and 6.5 [23]. However, for the majority of studies (17 of 18), gait disorders had to be established objectively before treatment by using the timed 25 -foot walk (T25FW). Certain studies (4 of 17) provided a precise time for this criterion: mean time to do the $\mathrm{T} 25 \mathrm{FW}$ was between 8 and $45 \mathrm{~s}[8,13,15,24]$. Only one study required patients to be able to walk for at least $3 \mathrm{~min}$ [20].

Exclusion criteria essentially corresponded to contraindications for fampridine, prior treatment with fampridine, and the existence of comorbidities. Concerning prior treatment with fampridine, it was considered an exclusion criterion in 2 of 18 studies. In the other studies, patients could be included provided that a wash-out period had been respected for at least $24 \mathrm{~h}$ [20] or, more prudently, for 4 weeks [16].

The existence of a flare-up at less than 60 days before inclusion $[8,9,14-19,24,25]$ was also an exclusion criterion. Similarly, patients that had a change in their diseasemodifying drug were excluded $[17,18,25,26]$.

\section{Principal Criterion}

The vast majority of studies (17 of 18) were based on analysis of walking speed using the T25FW, which is part of the multiple sclerosis functional composite (MSFC). The MSFC battery of tests also includes tests to evaluate upper-limb function (the 9 Hole-Peg Test) and cognition (the Paced Auditory Serial Addition Test). 


\section{Secondary Criteria}

Other walking tests, such as the 2-Minute Walk Test (2MWT) $[19,23,25,27]$ or the 6-Minute Walk Test (6MWT), were sometimes used in association with the T25FW to evaluate endurance $[16,20,28,29]$. The most recent studies $[17,18,28]$ also used the 6-Spot Step Test to evaluate coordination and balance while walking. Of these, 2 studies conducted an analysis of spatiotemporal gait parameters $[19,20]$.

A total of 9 studies completed objective analysis of gait subjectively by taking into account walking conditions in everyday life using the 12-item Multiple Sclerosis Walking Scale (MSWS-12) [8, 9, 14, 16, 19, 21, $24,27,28,30]$. This subjective evaluation of walking is specific to MS and includes 12 items scored on a 5-level Likert scale. The studies of Goodman et al. [8, 9] also assessed disease severity according to the opinions of both the patient and clinician using the clinician global impression and the subject global impression tests, which are scored on a Likert scale ranging from 1 to 7 .

The other MSFC scores mentioned above were also used as secondary criteria $[8,9]$.

Spasticity and muscle strength in the lower limbs were evaluated using the Ashworth scale and the Lower Extremity Manual Muscle Strength test, respectively [8, 9, $25,29]$.

Other symptoms of MS, such as fatigue and depression, were evaluated as secondary criteria using subjective scales $[19,23,30]$.

\section{Protocols}

With a cut-off of 28 days, 5 studies were classified as short term $[16-18,20,23]$ and 13 as long term $[8,9,14$, $15,19,21,22,24,25,27-30]$. The mean follow-up duration of short-term studies was 2.4 weeks and was 16 weeks for long-term studies.

All studies (including long-term studies) scheduled 2 visits (pre-treatment and post-treatment) with an interval of 2 weeks after initiation of treatment with fampridine in order to identify patients who had responded to treatment (Table 1). The majority of long-term studies also conducted an evaluation at 4 weeks after initiating treatment $[9,23,24,29,30]$.

Only the princeps studies of Goodman et al. $[8,9,14]$ used a protocol starting with 2 weeks of a placebo in a single-blind protocol before randomization into a placebo or a fampridine group. The study continued as a double-blind protocol for 14 weeks, period during which patients were evaluated 4 times.

MS and Clinical Gait Analysis before and after Fampridine
During these studies, the EDSS score was evaluated again $[8,9,14,16,22,30]$, and 2 studies used this score as a covariable to take into account disease progression [22, 30].

\section{Criteria for the Response to Fampridine}

Goodman et al. [8] reported that patients were deemed to have responded to fampridine if their walking speed had increased compared to the fastest of 4 speed evaluations made without treatment. This criterion was adopted by 7 of 18 studies $[8,9,14,15,24,28$, 29]. Pavsic et al. [23] deemed a patient had responded if walking speed in the T25FW had improved by $25 \%$ (compared with the first evaluation) after 14 days of treatment.

In 2 studies, evaluations were based on composite criteria. Allart et al. [19] used a composite criterion that associated 6 tests (T25FW, MSWS-12, 2MWT), with a significant improvement that was arbitrarily set at $15 \%$ in at least 1 of the 3 tests after 14 days of treatment. Prugger and Berger [30] conducted their study with several levels of response: (1) "the absence of any clinical benefit" corresponded to the absence of any improvement in the T25FW or in the MSWS-12 at 4 weeks; (2) a "moderate benefit" corresponded to an improvement of at least $10 \%$ in the T25FW and at least 6 points in the MSWS-12 at 4 weeks and 6 months; and (3) "a considerable benefit" corresponded to at least $20 \%$ improvement in the T25FW and at least 6 points in the MSWS-12 at 4 weeks or 6 months.

\section{Efficacy of Fampridine on Gait Disorders in MS}

\section{Patients}

\section{Principal Criterion}

Considering the principal criterion based on the $\mathrm{T} 25 \mathrm{FW}$, in the short term, the percentage improvement varied from study to study, ranging from 11.2 to $34.8 \%$. In the long term, the percentage improvement was the same order of magnitude, ranging from 10 to $40.1 \%$. (Fig. 2). Only one study, with a follow-up of $>1$ year, reported a precise figure for improvement in walking speed (20\%) compared to baseline values, but the difference was not significant [27]. The other studies used graphs to show improvements but did not indicate magnitudes [13, 28]. Ruck et al. [22]. showed that improvement in walking speed compared to baseline persisted for 9-12 months after initiating treatment, even after adjusting for disease progression, age, or EDSS score. In their 5-year study, Goodman et al. [14]. reported a decline in improvement in walking speed over time compared with baseline val- 


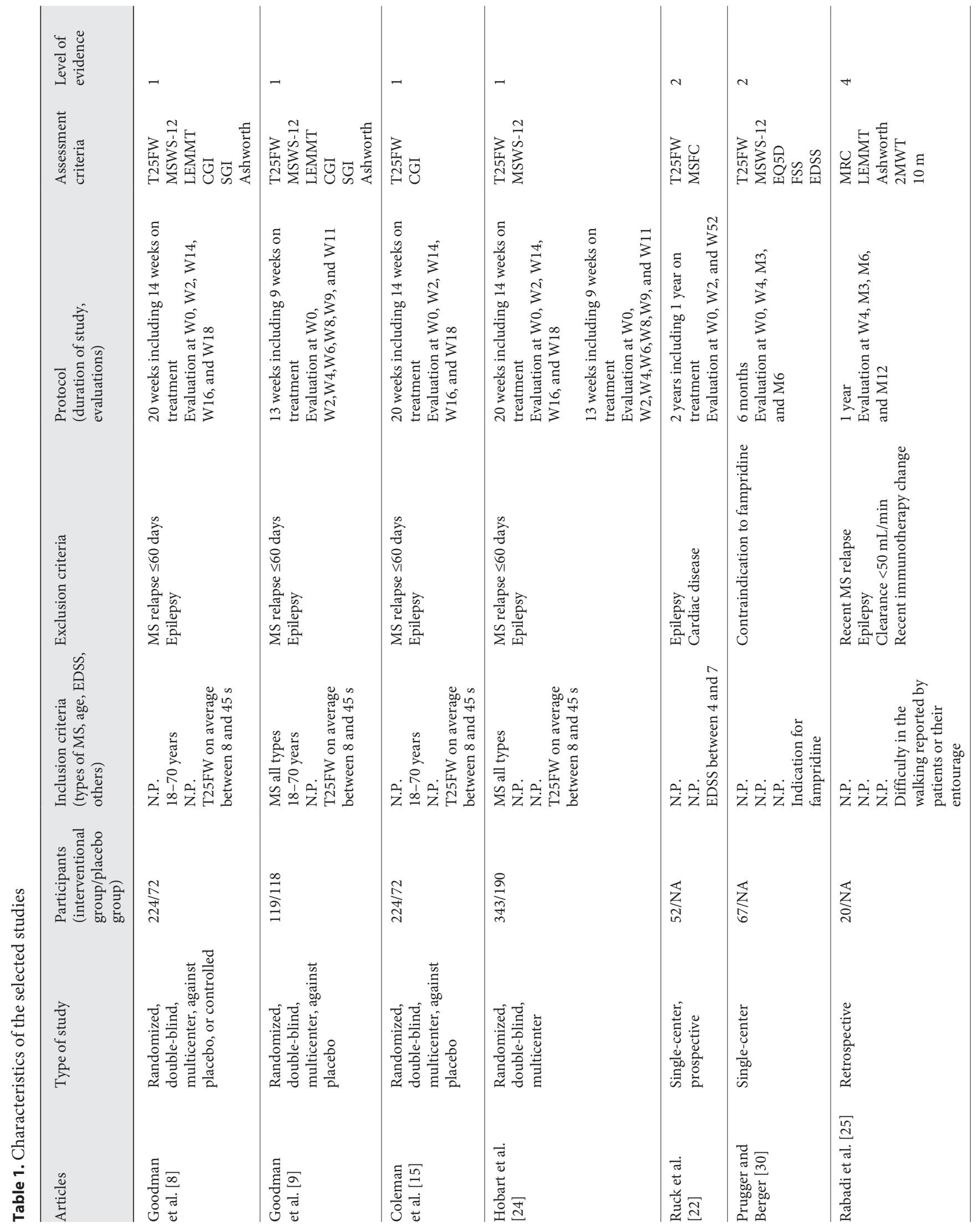




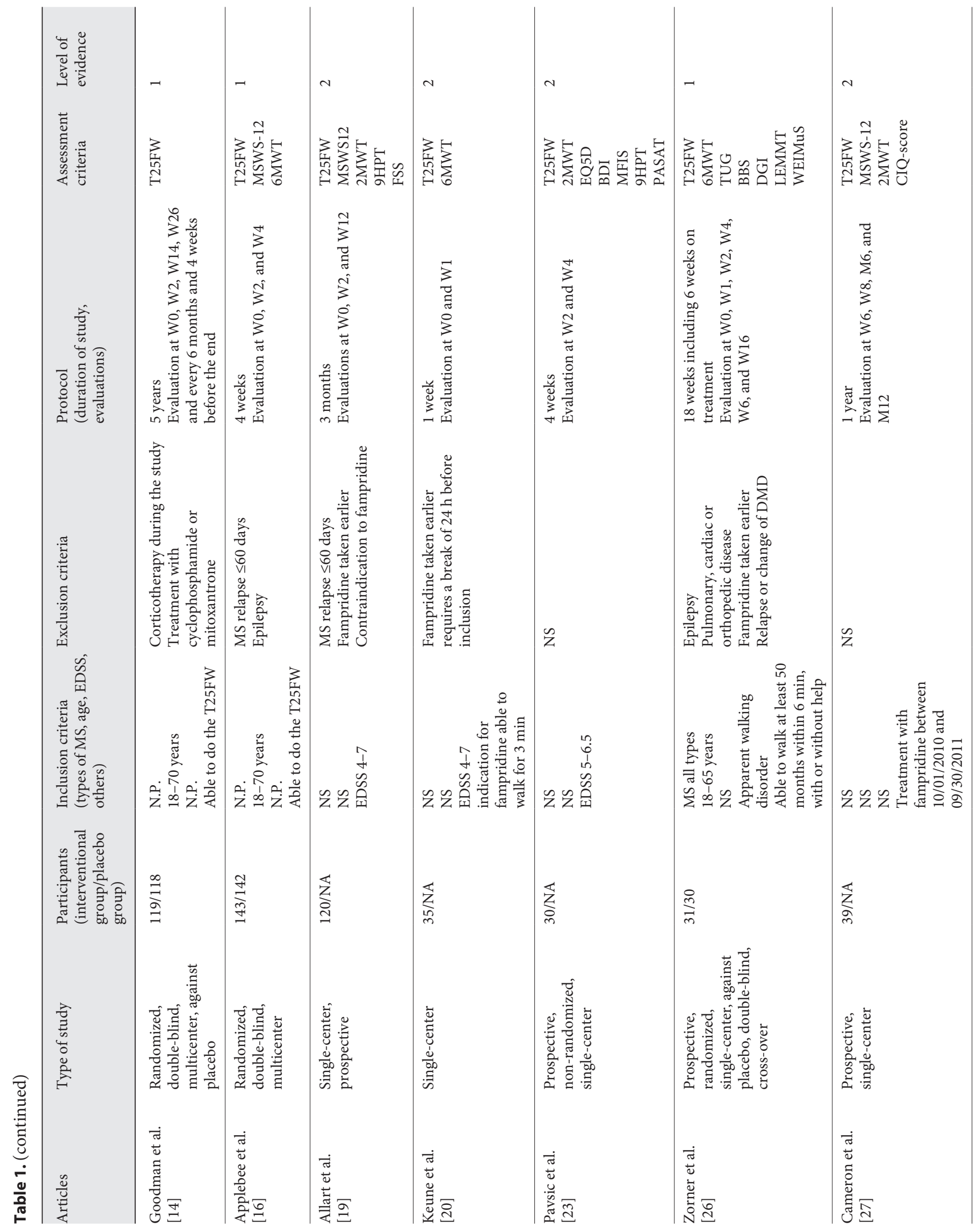




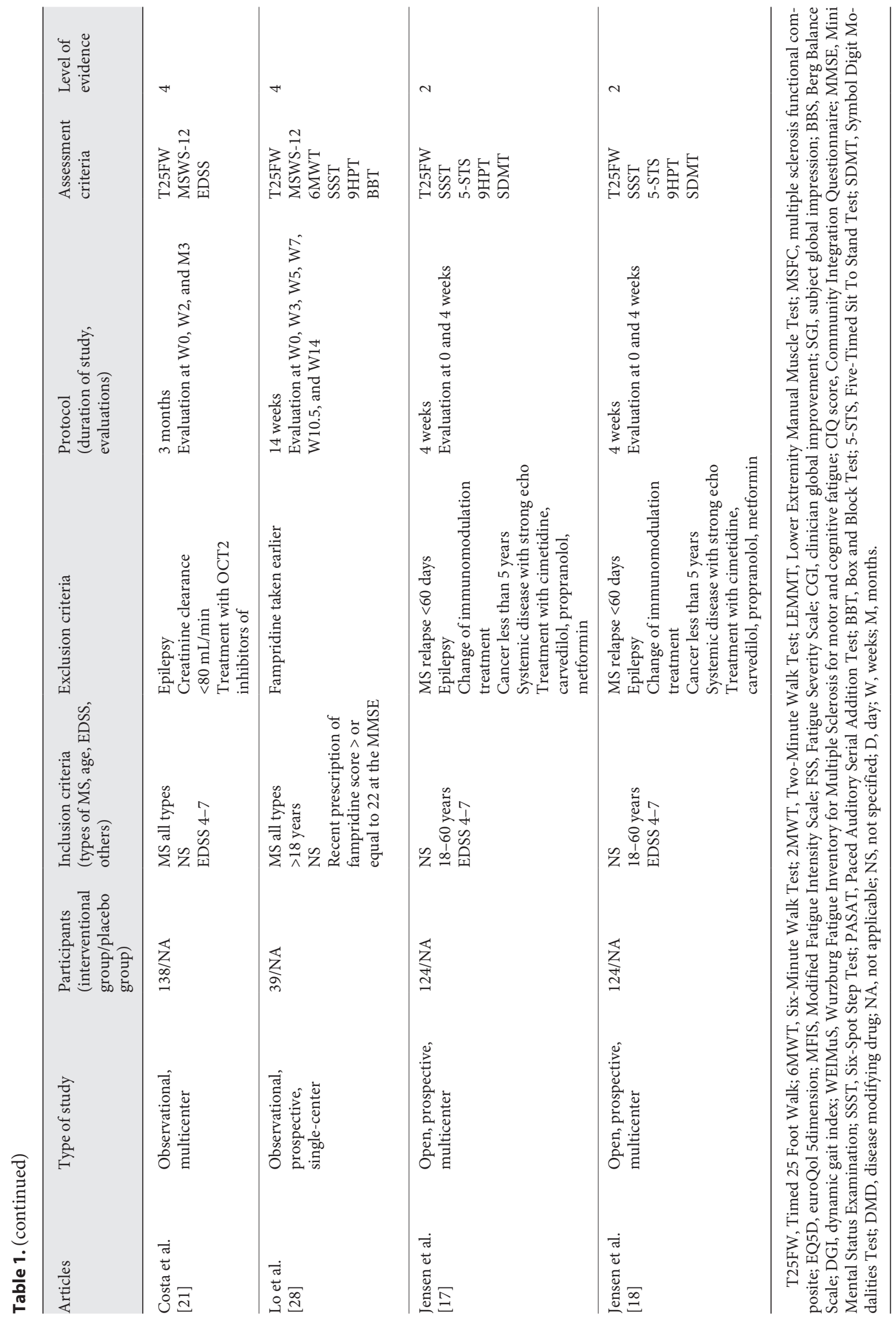




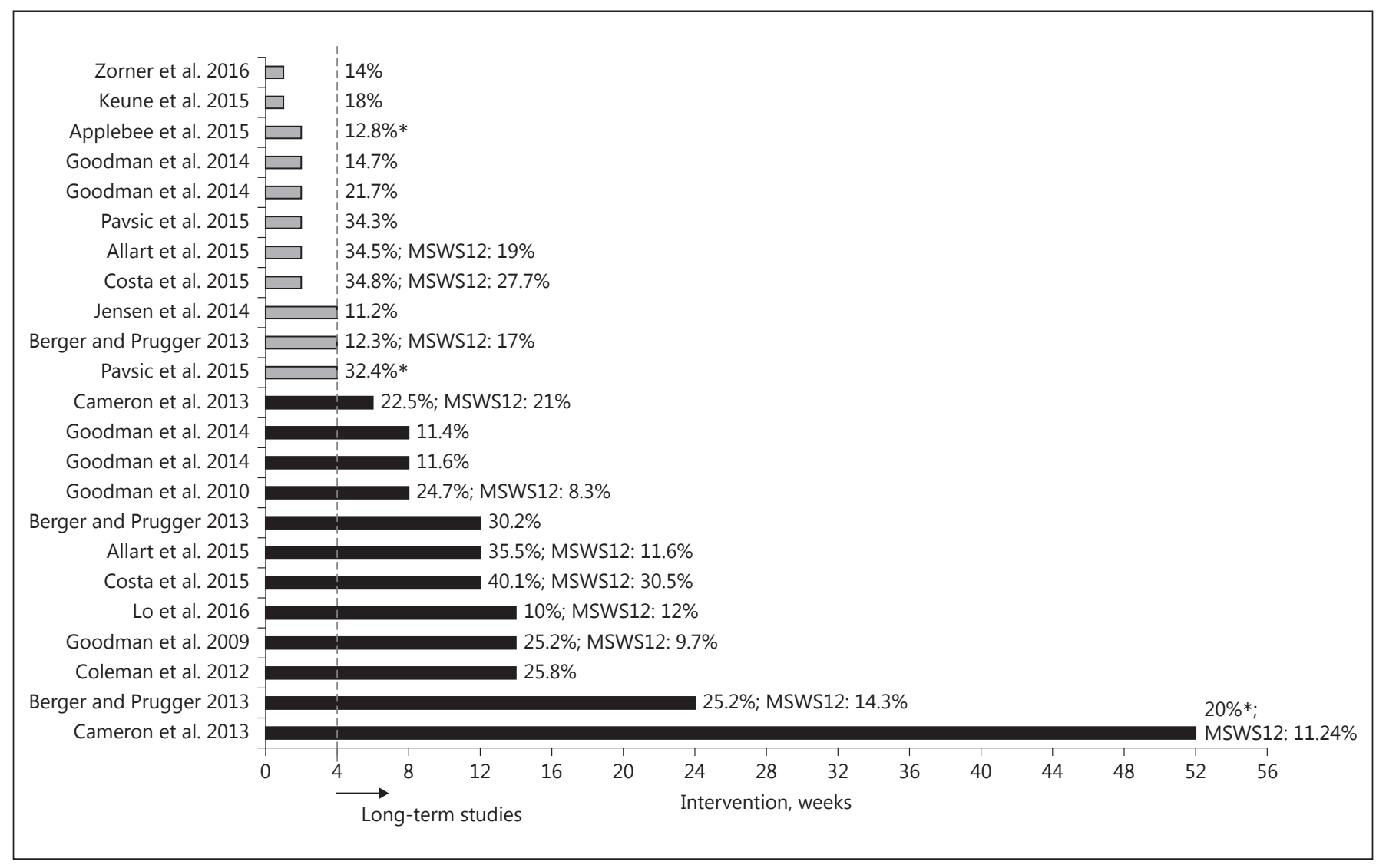

Fig. 2. Percentage improvement in the T25FW according to the study and according to the evaluation of the time point. * Non significative.

ues, although there was sustained improvement in responders compared to non-responders.

Between 31 and $74 \%$ of responders in the interventional group showed improvement following treatment with fampridine according to the principal judgement criterion $[8,9,14,19,23,28,29]$ compared to $8-9.3 \%$ in the placebo group [8, 9] (Table 2). The study by Allart et al. [19], based on a composite criterion but with an arbitrarily set threshold, showed a better response rate, that is, $74 \%$.

\section{Secondary Criteria}

Concerning the 2MWT, improvement ranged from 39 to $42.3 \%$ in the short term $[19,23,25,27]$ and was $36.7 \%$ at 3 months after initiating treatment [19]. For the 6MWT, the significant improvement in distance covered ranged from 8 to $23 \%$ in the interventional group $[16,20,29]$ compared to $4-12.2 \%$ in the placebo group $[16,29]$. The improvement in coordination (assessed by the 6-Spot Step Test) and the balance test ranged between 14 and $17 \%$ [18, 28] (Fig. 2).

MS and Clinical Gait Analysis before and after Fampridine
Keune et al. [20] conducted a study on spatiotemporal gait parameters; although fampridine increased walking and turning speed, these speeds diminished steadily during the $6 \mathrm{~min}$ of the test. This deceleration was significant before and after initiating fampridine treatment.

Concerning the MSWS-12 test, improvement in score ranged from 17 to $27.7 \%$ within the short term and from 8.3 to $30.5 \%$ within the long term. The correlation with the T25FW, as an absolute value, was weak ( $r=-0.2$ to $-0.3)$, but was very strong $(r=-0.33$ to -0.41$)$ when the variation in value was considered as a percentage $[19,24]$. An improvement in walking speed of at least $20 \%$ corresponded to an improvement in the MSWS-12 score of 6.4 points. One study also found a significant correlation between the results of the 2MWT and the MSWS-12 score [19] (Fig. 2).

Concerning the other studies, it is worth noting that significant improvements in walking speed and distance were not associated with any significant reduction in spasticity or improvement in muscle strength of the low- 


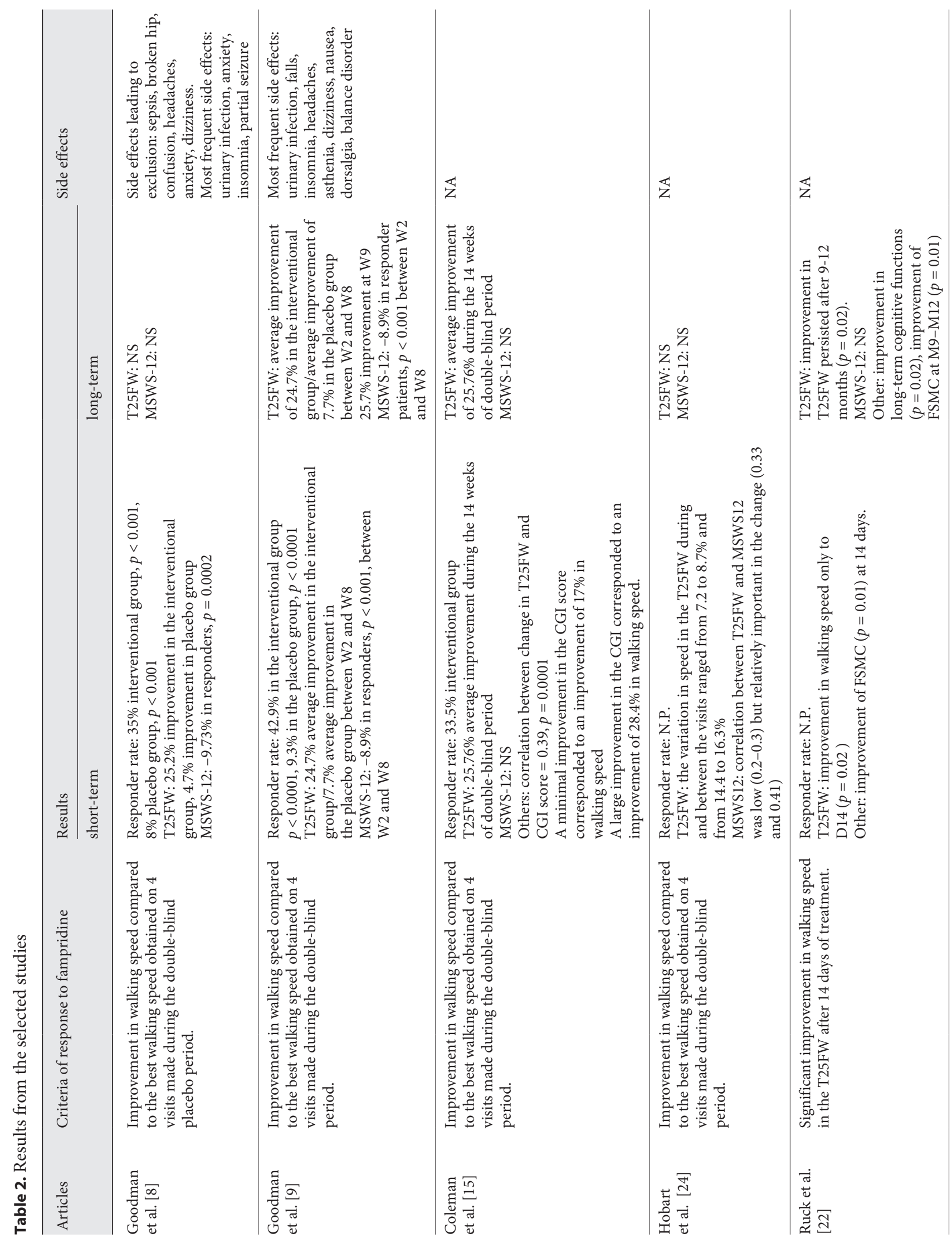




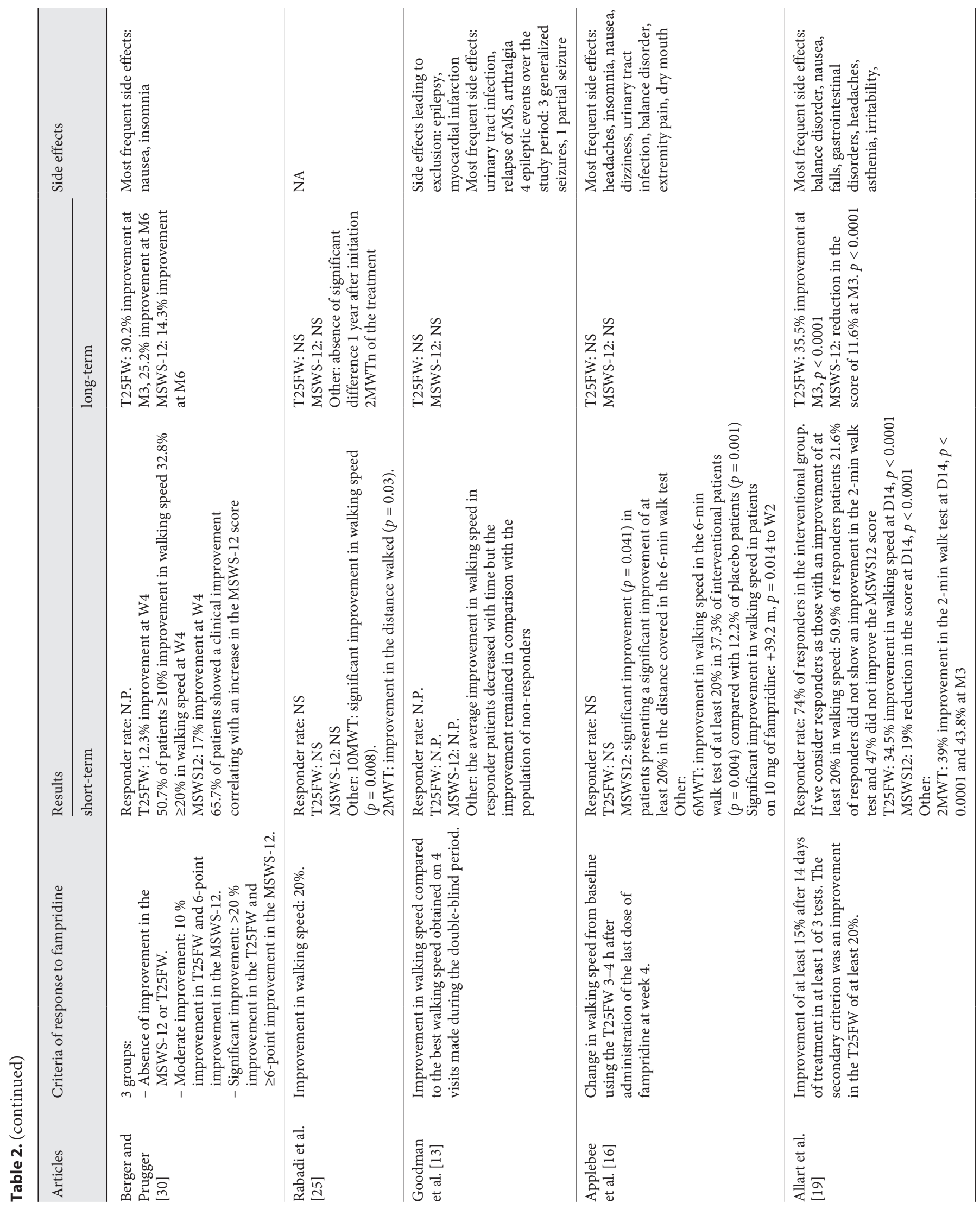



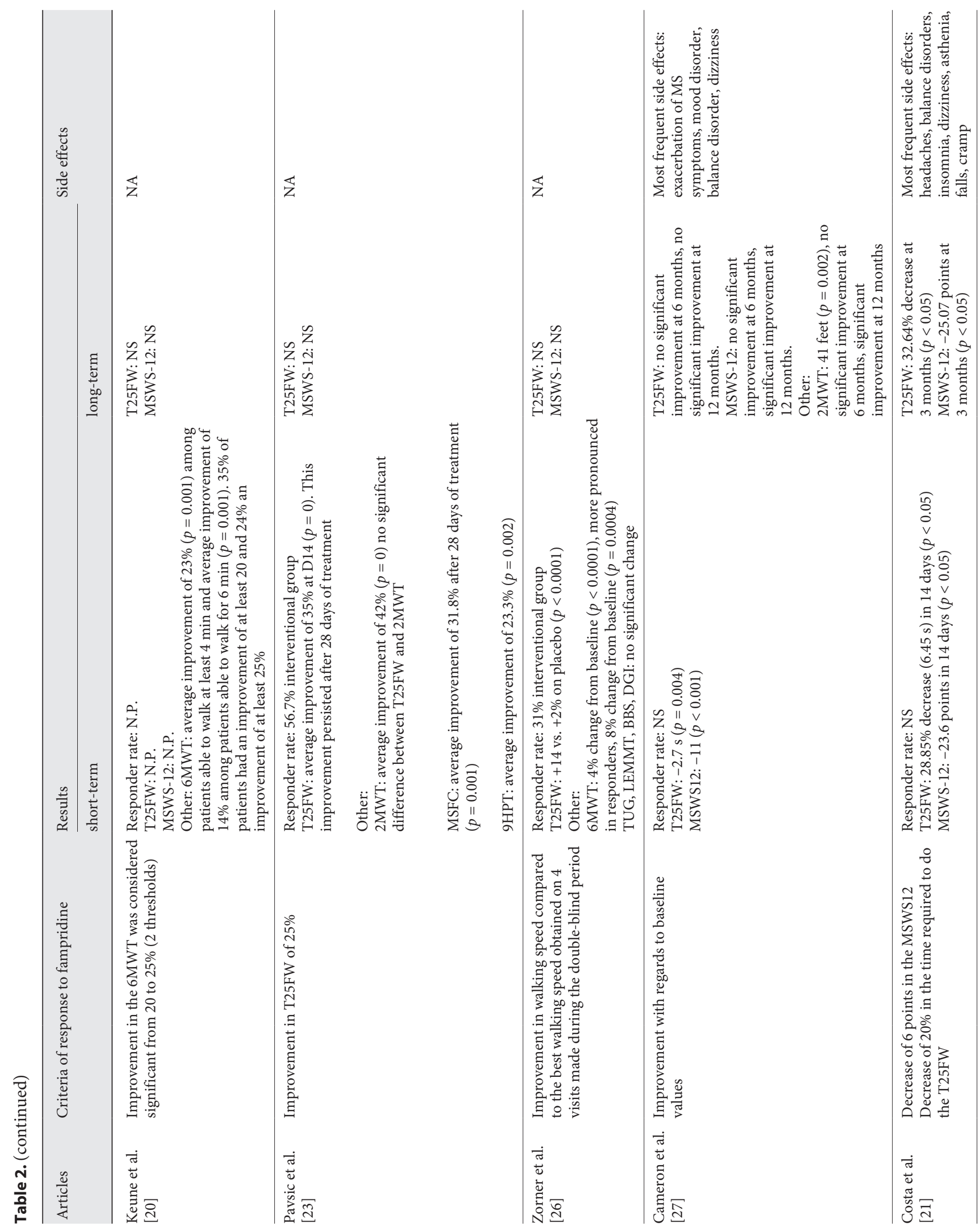


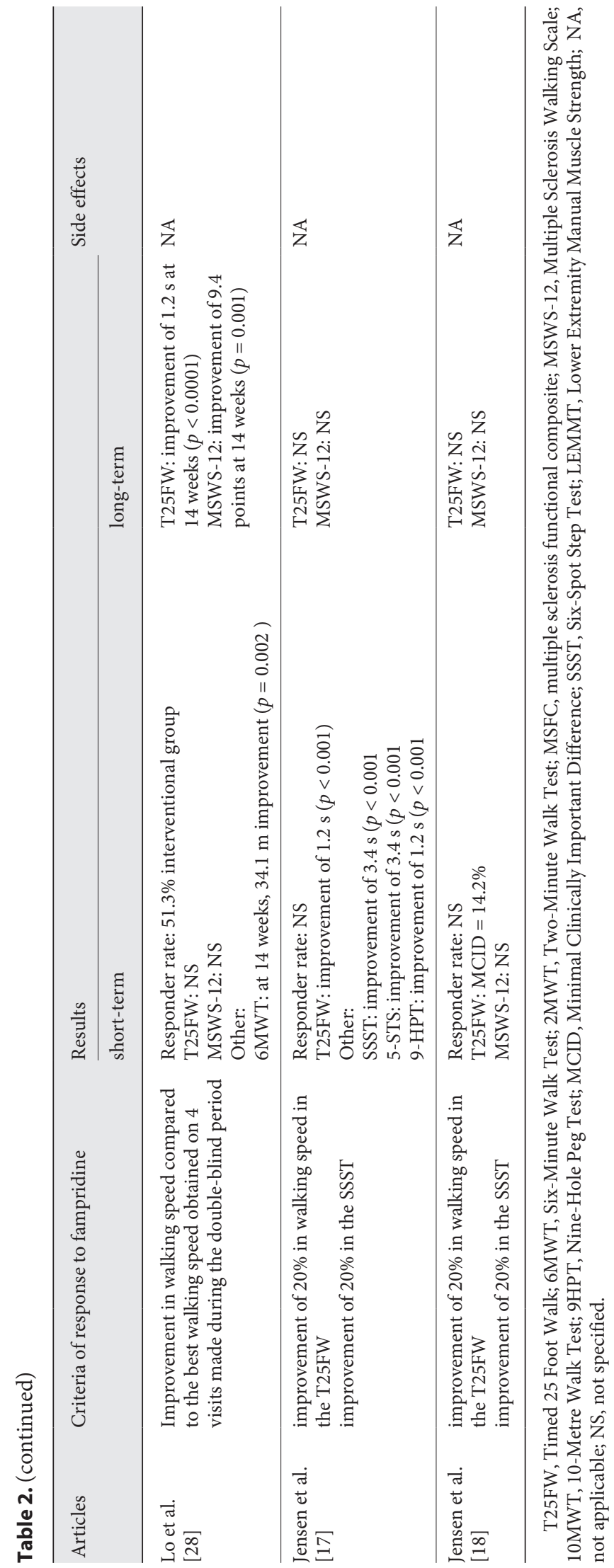

MS and Clinical Gait Analysis before and after Fampridine er limbs [25]. However, there was improvement in manual dexterity (9 Hole-Peg Test) in the short and long term in 3 studies [17, 23, 28]. One study reported improvement in cognitive function (Paced Auditory Serial Addition Test) in the long term (9-12 months after initiation of treatment) [23]. In addition, significant improvements in quality of life and fatigue were reported after 14 days and after 3 months of treatment [19].

The distance covered during the 6MWT improved by at least $20.3 \%$ in $37.3 \%$ of responders compared to $12.2 \%$ of the placebo group [16].

\section{Adverse Effects}

Less than half of the studies ( 8 of 18) investigated the adverse effects of fampridine $[8,9,14,16,19,21,27,30]$. Among these, 7 studies were long term, covering a period of 13 weeks to 5 years.

The most frequent adverse effects were urinary infections, falls, MS flare-ups, arthralgia, digestive disorders (nausea, constipation,..), headache, insomnia, asthenia, irritability, exacerbation of the existing neuropathy, and onset of peripheral edema. Only 3 patients presented with epilepsy, which was probably associated with the treatment [14]. One patient also presented with a severe adverse effect, myocardial infarction, which was probably related to the treatment [14].

\section{Discussion}

The aim of this literature review was to evaluate the short- and long-term efficacy and safety of fampridine. In MS, it is important to assess the long-term efficacy of this treatment, to define a profile of responder patients and a threshold of response, and to determine which walk tests to use in everyday practice. For this review, a total of 18 articles were included. The first study that focused on patients with MS and receiving fampridine dates back to 2009 [8].

In the light of this analysis, fampridine appears to be effective in the short term for patients with MS. Indeed, all of the identified studies found that 2 weeks of treatment with fampridine improved walking speed by between 11.2 and $34.8 \%$ in 21 and $74 \%$ of patients, respectively. The long-term analysis showed sustained improvement even though it was sometimes lower than the short-term threshold. For studies that lasted for $>1$ year, long-term progression was represented graphically with no additional information on the quantitative values [14, 25]. This made analysis difficult as it was difficult to distin- 
guish between the effect of disease progression, the impact of others factors than demyelination and that of decreased treatment efficacy. Moreover, at long term, gait disorders are not exclusively related to motor neurological disorders caused by demyelination [31]. Patients with MS are more prone to osteoarthritis [31] and there is a clear interaction between cognitive and gait disorders $[32,33]$.

Certain studies attempted to find correlations with the EDSS $[21,30]$ but this is also difficult because the EDSS score reflects both dimensions (evaluation and progression). Caution also needs to be exercised when interpreting improvement in walking speed, notably concerning the functional role of walking in everyday life. The percentage improvement in the $6 \mathrm{MWT}$ in responding patients was between 4 and $23 \%[16,20,29]$. When the different values in the different studies were compared, the percentage improvement in the experimental group of one study [29] was less than the improvement in the placebo group in another study [16]. This shows that the notion of percentage improvement in the absence of a significant threshold for change is difficult to interpret, all the more so as there is no consensus regarding a threshold for a response.

Overall, these studies show that there were no predictive factors for a good response in the population of MS patients. One study reported a non-significant trend in patients with a progressive form of MS [27]. This could be partly explained by the over-representation of these patients, as they accounted for the majority of patients included. In addition, these patients presented with more severely impaired gait, and thus showed a better margin for percentage improvement for the same absolute value. Although the number of studies was relatively small, there were reports of other patient-related factors that may have led to the variability in response to treatment, such as the location of the lesions, or genetic differences with regards to potassium channels [30,34].

Even though the majority of studies used the T25FW, there was some difficulty in determining a threshold to identify a patient as a responder. This test is part of the MSFC and earlier studies have demonstrated the validity and sensitivity of a $20 \%$ threshold, above which changes were greater than normal variations in individuals [35-38]. This has proved to be ideal for "slow" patients [19], where a small absolute improvement leads to a large percentage improvement. However, in "fast" patients, the percentage variation in this test is not representative and does not seem to be particularly sensitive at detecting changes. In this systematic review, longer tests (2MWT and 6MWT) appeared to be more relevant to assess endurance $[16,19,25]$ and more sensitive at detecting the effects of fampridine [14], and had lower variability. These findings were suggested in earlier studies, which also reported that long-term tests were more sensitive at detecting improvements after rehabilitation and reflected everyday life activities more effectively $[39,40]$. In order to consider the different types of gait impairment (impaired balance, spastic gait,...), Allart et al. [19] used a composite criterion including upper limb evaluation and an arbitrary threshold of a 15\% improvement and found a response rate of $74 \%$. This composite strategy should be more appropriate and should take into account for future studies as it permits to assess the improvements of patients with a fast or slow walking speed (EDSS $>7$ ) at the moment the treatment begins.

The majority of studies identified patients as responders to fampridine if there was an improvement in walking speed in the T25FW at 2 weeks after initiating treatment compared to the fastest walking speed obtained without treatment. Other studies were based on a minimal $20 \%$ improvement in walking speed $[17,30]$. However, Hobart et al. [24] argued that the relatively low thresholds did not take into account the considerable variability related to the T25FW. Indeed, $75 \%$ of patients showed improved walking speed of at least $10 \%$, but most measurements (75\%) done in the different visits varied by around $24 \%$. This percentage corresponds to those reported in previous studies [35, 36]. Moreover, regarding other studies, there is considerable variability during the same day especially for the T25FW (31\%) [41].

The minimal thresholds to detect a change in spatiotemporal gait parameters were determined and compared with those obtained using detection thresholds, such as a $17.2 \%$ improvement in walking speed [15].

In most studies, patients receiving fampridine before the start of the study could be included provided there was a 24 -h wash-out period beforehand. This interval could be rather short given that a wash-out of 2 weeks in cross-over studies has been deemed necessary to eliminate any effect (direct and indirect) linked to fampridine $[8,9,14]$. Moreover, given that this drug is eliminated almost exclusively by the kidneys, a pharmacokinetics analysis revealed that, in $24 \mathrm{~h}$, around $90 \%$ of the dose was eliminated in an unaltered form in the urine. What is more, there may be an indirect effect of treatment-related improvement, as the improvement in motor function engenders a global improvement in other structures and functions [42], even if the molecular action of the treatment, given its half-life of $6 \mathrm{~h}$, should disappear at $\sim 42 \mathrm{~h}$ 
after taking it [6]. Thus, to be sure that fampridine has been eliminated, a wash-out period of at least $48 \mathrm{~h}$ seems necessary. The results of studies that did not respect this interval should therefore be interpreted with caution, as there could be a persistent effect from prior administration of fampridine, which would be less noticeable at the second measurement [20].

In most studies, the T25FW test was associated with a subjective test on the perception of the impact of the disease on everyday activities (MSWS-12). This reflects the difficulty in establishing a single criterion to evaluate the effect of fampridine on walking in MS patients. The improvement in the MSWS-12 score ranged from 8 to $30 \%$ in the short and long term, and given that this test correlated with the T25FW, a $20 \%$ mean improvement in walking speed corresponded to an improvement of 6.4 points in the MSWS-12 score [24]. However, numerous sources of bias related to these subjective criteria, and notably due to cognitive impairment and memory disorders found in MS, make it difficult to interpret these results.

Adverse effects were relatively frequent but were generally mild and transient; very few adverse effects led to treatment cessation in the short or long term $[9,14]$ and in all of the studies combined, only 4 severe adverse events occurred. The most frequently reported adverse effects were headache and dizziness. Episodes of epilepsy were rare but expected given the reduced epileptogenic threshold. Concerning urinary symptoms, most of the studies reported detrusor overactivity. This is in line with a previous study showing that under fampridine treatment only $30 \%$ of patients with urinary symptoms have an infection [43].

Although a previous study found a relationship between efficacy/adverse effects and the blood level of fampridine [44], only one study [13] investigated inter-dose effect showing that a $25 \%$ decrease in the concentration of fampridine did not correlate with decreased walking speed. The other studies considered in this review did not investigate this topic.

This systematic review has several limitations, the first being the exclusion of articles written in languages other than English, even though there were only 5 articles in this category. Another limitation was the small number of articles, which meant that they were analyzed independently of the LE to ensure that the analysis was as complete as possible.

Given the relatively recent approval of fampridine, this study could be continued by conducting an updated study. Moreover, consultation of the registration website for clinical trials (clinicaltrials.gov) shows that 10 studies on this topic are ongoing and 2 have recently been completed (2016).

\section{Conclusion}

This systematic review provides additional evidence that fampridine increases walking speed in the shortterm in patients with MS. This treatment is also valid in the longer term, that is to say up to 1 year. However, given the small number of studies and the disparity between them, it is not possible to draw any conclusions on treatment efficacy beyond 1 year. The drug seems to be well tolerated as the adverse effects in the short and long term were mild and transient.

Concerning the evaluation, all of the selected studies analyzed gait over short distances, but other evaluations of gait could be conducted, thus widening the scope of assessing human locomotion. Thus, it seems necessary to conduct long-term studies that can evaluate other gait parameters, notably objective evaluations in real-life settings of patients taking fampridine. Studies on a return to other activities, whether in rehabilitation or not, could also add to these data.

\section{Disclosure Statement}

The authors declare no conflicts of interest.

\section{References}

1 Larocca NG: Impact of walking impairment in multiple sclerosis: perspectives of patients and care partners. Patient 2011;4:189-201.

2 Yildiz M: The impact of slower walking speed on activities of daily living in patients with multiple sclerosis. Int J Clin Pract 2012;66: 1088-1094.

3 Hobart JC, Riazi A, Lamping DL, Fitzpatrick $\mathrm{R}$, Thompson AJ: Measuring the impact of MS on walking ability: the 12-Item MS Walking Scale (MSWS-12). Neurology 2003;60: 31-36.

4 Créange A, Serre I, Levasseur M, Audry D, Nineb A, Boërio D, et al: Walking capacities in multiple sclerosis measured by global positioning system odometer. Mult Scler 2007;13: 220-223.

5 Pike J, Jones E, Rajagopalan K, Piercy J, Anderson P: Social and economic burden of walking and mobility problems in multiple sclerosis. BMC Neurol 2012;12:94.

6 FAMPYRA-Product-Monograph19Dec2012-Fr.pdf. 
7 Hayes KC: Fampridine-SR for multiple sclerosis and spinal cord injury. Expert Rev Neurother 2007;7:453-461.

8 Goodman AD, Brown TR, Krupp LB, Schapiro RT, Schwid SR, Cohen R, et al: Sustainedrelease oral fampridine in multiple sclerosis: a randomised, double-blind, controlled trial. Lancet 2009;373:732-738.

9 Goodman AD, Brown TR, Edwards KR, Krupp LB, Schapiro RT, Cohen R, et al: A phase 3 trial of extended release oral dalfampridine in multiple sclerosis. Ann Neurol 2010;68:494-502.

10 Shamseer L, Moher D, Clarke M, Ghersi D, Liberati A, Petticrew M, et al: Preferred reporting items for systematic review and meta-analysis protocols (PRISMA-P) 2015: elaboration and explanation. BMJ 2015; 349:g7647.

11 Moher D, Stewart L, Shekelle P: Implementing PRISMA-P: recommendations for prospective authors. Syst Rev 2016;5:15.

12 Lublin FD, Reingold SC, Cohen JA, Cutter GR, Sørensen PS, Thompson AJ, et al: Defining the clinical course of multiple sclerosis: the 2013 revisions. Neurology 2014;83:278286.

13 Goodman AD, Brown TR, Cohen JA, Krupp LB, Schapiro R, Schwid SR, et al: Dose comparison trial of sustained-release fampridine in multiple sclerosis. Neurology 2008;71: 1134-1141.

14 Goodman AD, Bethoux F, Brown TR, Schapiro RT, Cohen R, Marinucci LN, et al: Longterm safety and efficacy of dalfampridine for walking impairment in patients with multiple sclerosis: Results of open-label extensions of two Phase 3 clinical trials. Mult Scler 2015;21: 1322-1331.

15 Coleman CI, Sobieraj DM, Marinucci LN: Minimally important clinical difference of the timed 25-foot walk test: results from a randomized controlled trial in patients with multiple sclerosis. Curr Med Res Opin 2012;28: 49-56.

16 Applebee A, Goodman AD, Mayadev AS, Bethoux F, Goldman MD, Klingler M, et al: Effects of dalfampridine extended-release tablets on 6-minute walk distance in patients with multiple sclerosis: a post hoc analysis of a double-blind, placebo-controlled trial. Clin Ther 2015;37:2780-2787.

17 Jensen H, Ravnborg M, Mamoei S, Dalgas U, Stenager E: Changes in cognition, arm function and lower body function after slow-release Fampridine treatment. Mult Scler 2014; 20:1872-1880.

18 Jensen HB, Mamoei S, Ravnborg M, Dalgas U, Stenager E: Distribution-based estimates of minimum clinically important difference in cognition, arm function and lower body function after slow release-fampridine treatment of patients with multiple sclerosis. Mult Scler Relat Disord 2016:7:58-60.
19 Allart E, Benoit A, Blanchard-Dauphin A, Tiffreau V, Thevenon A, Zephir H, et al: Sustained-released fampridine in multiple sclerosis: effects on gait parameters, arm function, fatigue, and quality of life. J Neurol 2015; 262:1936-1945

20 Keune PM, Cocks AJ, Young WR, Burschka JM, Hansen S, Hofstadt-van Oy U, et al: Dynamic walking features and improved walking performance in multiple sclerosis patients treated with fampridine (4-aminopyridine). BMC Neurol 2015;15:171.

21 Costa-Arpín E, Pato A, Rodríguez-Regal A, Midaglia L, Yáñez R, Muñoz D, et al: Clinical response and tolerability of fampridine in clinical practice. Neurodegener Dis Manag 2016;6:99-105.

22 Ruck T, Bittner S, Simon OJ, Göbel K, Wiendl $\mathrm{H}$, Schilling M, et al: Long-term effects of dalfampridine in patients with multiple sclerosis. J Neurol Sci 2014;337:18-24.

23 Pavsic K, Pelicon K, Ledinek AH, Sega S: Short-term impact of fampridine on motor and cognitive functions, mood and quality of life among multiple sclerosis patients. Clin Neurol Neurosurg 2015;139:35-40.

24 Hobart J, Blight AR, Goodman A, Lynn F, Putzki N: Timed 25-foot walk: direct evidence that improving $20 \%$ or greater is clinically meaningful in MS. Neurology 2013;80:15091517.

25 Rabadi MH, Kreymborg K, Vincent AS: Sustained-release fampridine (4-aminopyridine) in multiple sclerosis: efficacy and impact on motor function. Drugs R D 2013;13:175-181.

26 Zörner B, Filli L, Reuter K, Kapitza S, Lörincz L, Sutter T, et al: Prolonged-release fampridine in multiple sclerosis: Improved ambulation effected by changes in walking pattern. Mult Scler 2016; 22:1463-1475.

27 Cameron MH, Fitzpatrick M, Overs S, Murchison C, Manning J, Whitham R: Dalfampridine improves walking speed, walking endurance, and community participation in veterans with multiple sclerosis: a longitudinal cohort study. Mult Scler 2014;20:733-738.

28 Lo AC, Ruiz JA, Koenig CM, Anderson BM, Olson KM, Triche EW: Effects of dalfampridine on multi-dimensional aspects of gait and dexterity in multiple sclerosis among timed walk responders and non-responders. J Neurol Sci 2015;356:77-82.

29 Zörner B, Filli L, Reuter K, Kapitza S, Lörincz L, Sutter T, et al: Prolonged-release fampridine in multiple sclerosis: improved ambulation effected by changes in walking pattern. Mult Scler 2016;22:1463-1475.

30 Prugger M, Berger T: Assessing the long-term clinical benefit of prolonged-release fampridine tablets in a real-world setting: a review of 67 cases. Patient Relat Outcome Meas 2013;4. 75-85.

31 Eroglu S, Inal EE, Eroglu M, Oruc S, Ulasli $\mathrm{AM}$, Cevik $\mathrm{H}$, et al: Ultrasound detection of knee joint degeneration in patients with multiple sclerosis. J Rehabil Med 2016;48:604608

32 Allali G, Laidet M, Assal F, Armand S, Lalive $\mathrm{PH}$ : Walking while talking in patients with multiple sclerosis: the impact of specific $\operatorname{cog}$ nitive loads. Neurophysiol Clin 2014;44:8793.

33 Downer MB, Kirkland MC, Wallack EM, Ploughman M: Walking impairs cognitive performance among people with multiple sclerosis but not controls. Hum Mov Sci 2016; 49:124-131.

34 Goodman AD, Stone RT: Enhancing neural transmission in multiple sclerosis (4-aminopyridine therapy). Neurotherapeutics 2013; 10:106-110.

35 Kaufman M, Moyer D, Norton J: The significant change for the Timed 25-foot walk in the multiple sclerosis functional composite. Mult Scler 2000;6:286-290.

36 Schwid SR, Goodman AD, McDermott MP, Bever CF, Cook SD: Quantitative functional measures in MS: what is a reliable change? Neurology 2002;58:1294-1296.

37 Kragt JJ, Linden van der FA, Nielsen JM, Uitdehaag BM, Polman CH: Clinical impact of $20 \%$ worsening on timed 25 -foot walk and 9-hole Peg Test in multiple sclerosis. Mult Scler 2006;12:594-598.

38 Hoogervorst EL, Kalkers NF, Cutter GR, Uitdehaag BM, Polman $\mathrm{CH}$ : The patient's perception of a (reliable) change in the multiple sclerosis functional composite. Mult Scler 2004; 10:55-60.

39 Goldman MD, Marrie RA, Cohen JA: Evaluation of the six-minute walk in multiple sclerosis subjects and healthy controls. Mult Scler 2008; 14:383-390.

40 Savci S, Inal-Ince D, Arikan H, Guclu-Gunduz A, Cetisli-Korkmaz N, Armutlu K, et al: Six-minute walk distance as a measure of functional exercise capacity in multiple sclerosis. Disabil Rehabil 2005;27:1365-1371.

41 Feys P, Bibby B, Romberg A, Santoyo C, Gebara B, de Noordhout BM, et al: Within-day variability on short and long walking tests in persons with multiple sclerosis. J Neurol Sci 2014;338:183-187.

42 Studenski S, Perera S, Patel K, Rosano C, Faulkner K, Inzitari M, et al: Gait speed and survival in older adults. J Am Med Assoc 2011;305:50-58.

43 Kantor D, Chancellor MB, Snell CW, Henney HR 3rd, Rabinowicz AL: Assessment of confirmed urinary tract infection in patients treated with dalfampridine for multiple sclerosis. Postgrad Med 2015;127:218-222.

44 Van Diemen HA, Polman CH, Koetsier JC, Van Loenen AC, Nauta JJ, Bertelsmann FW: 4-Aminopyridine in patients with multiple sclerosis: dosage and serum level related to efficacy and safety. Clin Neuropharmacol 1993; 16:195-204. 